\title{
Motion, Energy and State of Body Particle System
}

\author{
Zhong-Cheng Liang \\ School of Electronic and Optical Engineering, Nanjing University of Posts and Telecommunications, Nanjing, China \\ E-mail: zcliang@njupt.edu.cn
}

\begin{abstract}
Based on the assumption that protons and electrons are body particles, the motion and state of body particle system are investigated in an energy space. Body particles are objects that have only mass and volume. A body particle has three spatial states: position, posture and profile, corresponding to three modes of motion: translation, rotation and vibration. The energies of three modes of particle system constitute a Descartes energy space. The state of object is represented by a state vector in the energy space. The energy space can be divided into three zones and six phases. The three zones dominated by translation, rotation and vibration modes represent the liquid, solid and gaseous state of the object, respectively. There are three parabolic surfaces in energy space, which represent the equilibrium states of the particle system. It is showed that there are two types of phase transitions in energy space, corresponding to that the change of order parameter is equal to $1 / 2$ or less than $1 / 2$. There are a stable equilibrium area and an unstable equilibrium area in each phase, which predicts the transition between the stable state and the excited state within the phase. Strict analysis proves that the number of energy in equilibrium state must be integers. The Planck constant and Boltzmann constant are both scale transformation coefficients of the motion energy.
\end{abstract}

Keywords: Particle, motion, energy, state transition, phase transition, quantum

\section{Introduction}

Particle is a fundamental concept of physics. Different particle models lead to different physical theories. Classical physics regards particles as zero-dimensional point particles, which occupy no space. Quantum physics assumes that particles are matter waves, which occupy space but have no definite volume. In current physics, the interaction between particles is imposed on the particles through the assumptions such as mass, charge, spin and symmetry, without explaining their origins. Different particle models need different methods to describe the state of particles, so as to express the laws of motion in different formulations. Classical mechanics uses trajectories to describe the motion of point mass, and the motion of particles is determined by Newtonian laws [1]. Electrodynamics conceives that charged particles excite the surrounding electromagnetic field, and the motion of charged particles is controlled by Maxwell field equations [2]. Wave mechanics in quantum physics uses wave functions to describe the state of microscopic particles. The change of state is governed by Schrodinger's equation [3]. A logically consistent theory may have different representations, but they are essentially equivalent. For examples, vector mechanics and analytical mechanics of classical physics are equivalent, and wave mechanics and matrix mechanics of quantum physics are equivalent. However, classical mechanics, electrodynamics and quantum mechanics belong to unequal theoretical systems because of their different models of particles and interactions.

Three years ago, the author of this article proposed a physical theory based on the model of body particles [4]. Body particles are three-dimensional objects that have only mass and volume. Body particle is a model essentially different from the point particle and the matter wave. The fundamental feature is that the body particles have the interaction of volume repulsion, namely, different particles cannot overlap in space at the same time. In contrast, point particles can be infinitely close to each other in space, and the interaction between particles is determined by properties such as mass and charge. Although wave functions occupy space, the wave functions of different particles can overlap in space at the same time. Unlike particle trajectories in classical mechanics and wave functions in quantum mechanics, body particle theory describes the motion of particles and the state of objects through discrete sets of position vectors.

For an object with finite volume composed of a large number of particles, the mass and momentum of the particles can be counted in discrete spatial grids. By constructing the mass and momentum potentials, a complete set of particle field equations can be derived to explain the origin of gravitational and 
electromagnetic interaction [4,5]. Body particle theory shows that the field is a statistical phenomenon of a huge number of particles, and reveals that the physics should actually be a theory of particle statistics. The theory of particle field is based on the statistics of mass and momentum, and the theory of state of object is based on the statistics of particle energy. This article introduces the theory of state of object, and investigates the properties of state transition of the particle system.

\section{Concepts and Principles}

As a systematic theory different from both classical and modern physics, it is necessary to reinspect primitive concepts and introduce new principles $[4,5]$.

\subsection{Principle of Object Structure}

(1) Object. An object is three-dimensional entity that has only mass and volume.

(2) Particle. A particle is three-dimensional entity that has only mass and volume. Such particle is termed body particle (or entity particle [5]), in contrast to the point particle.

(3) Axiom of object structure (Axiom 1). Any object is composed of particles with nesting structure. An object can be decomposed into discrete and finite particles.

(4) Primary particle. A primary particle is indivisible particle that has constant mass.

(5) Axiom of primary particle (Axiom 2). There are only two types of primary particles of different mass: proton and electron.

(6) Theorem of mass imperishability. Mass is an inherent property of particles, and the mass of a primary particle is constant. All objects are made up of primary particles, so the mass of an object cannot be annihilated.

\subsection{Principles of Space, Time and Motion}

(1) Space. Space is the place in which the objects exist and move. The real space is continuous, uniform and three-dimensional.

(2) Axiom of real space (Axiom 3). Real space is filled with particles. There exists no empty space that has no particles.

(3) Time. Time is a progression with which the objects exist and move. The time is continuous, uniform and unidirectional.

(4) Motion. Motion is the process that the state of object changes with time in space.

(5) Volume. The volume of an object is the space required for the motion of its internal particles.

(6) Theorem of volume repulsion. The motion of particles requires space and time. The volume of different particles does not intersect each other in real space at the same time.

(7) Real physics. Real physics is a theory based on real space and restricted in the domain (field) of real number.

\subsection{Principle of Objectivity}

(1) Physical quantity. Any physical quantity $\boldsymbol{x}$ can be decomposed as the product of scale $x_{s}$ and digit $\tilde{\boldsymbol{x}}$.

$$
\boldsymbol{x}=x_{s} \cdot \widetilde{\boldsymbol{x}}=x_{s} \times \widetilde{\boldsymbol{x}} ; x_{s}>0 .
$$

The scale is the unit of measurement and the identification of the physical quantity, while the digit is the number of the physical quantity.

The scale is a scalar in the domain of positive real number. Depending on the type of physical quantity, the digit can be a scalar, a vector or a tensor. For examples, the digit of time $(\tilde{t})$ is scalar, and the digits of position and velocity $(\tilde{\boldsymbol{r}}, \widetilde{\boldsymbol{u}})$ are vectors.

(2) Objective and subjective quantity. The physical quantity $\boldsymbol{x}$ is objective quantity, and the scale $x_{s}$ is subjective quantity.

(3) Absolute and relative quantity. The physical quantity $\boldsymbol{x}$ is absolute quantity, and the digit $\tilde{\boldsymbol{x}}$ is relative quantity. 
(4) Axiom of objectivity (Axiom 4). The physical quantities are objective and the laws of motion are objective. Physical theory must exclude all subjective factors. The objectivity axiom requires the object measurability, the origin irrelevance, and the scale irrelevance.

(5) Object measurability. Objects are measurable. A measurable quantity is finite and has scale with $x_{s}>0$. Object measurability excludes the infinity $( \pm \infty)$ from physics.

(6) Origin irrelevance. The reference points of space and time are subjective and relative. All physical formulas must be independent of the origins of space and time.

(7) Scale irrelevance. Any physical relation $z=R(x, y)$ is independent of the scales, i.e. the physical relation and digital relation are equivalent, namely

$$
z=R(x, y)=z_{s} \cdot \tilde{z} ; \quad \tilde{z}=R(\tilde{x}, \tilde{y}) .
$$

The scale formula (1) is the basis for discretization and digitization of physical quantity. The axiom of objectivity is essentially the principle of universality. The origin irrelevance means that the physical laws are applicable to any place and any time. The scale irrelevance means that the physical laws are applicable to any scale. For example, there should be no difference between microphysics and macrophysics.

\section{$3 \quad$ Structure and State}

Discrete mathematics is the logic tool for dealing with dispersed particle systems. The basis of discrete mathematics is the set theory. In this article, set symbols are represented by double-line font.

\subsection{Structure of Object}

If electron is represented by symbol 0 and proton by symbol 1, any object can be represented by a set consisting of 0 and 1 .

(a) Electron. The mass of an electron is $M_{e}=9.1093821 \times 10^{-31} \mathrm{~kg}$. The set of $a$ electrons is represented as

$$
\mathbb{E}^{a}=\underbrace{\{0,0,0, \cdots, 0\}}_{a} .
$$

(b) Proton. The mass of an proton is $M_{p}=1.6726216 \times 10^{-27} \mathrm{~kg}$. The set of $b$ protons is represented as

$$
\mathbb{P}^{b}=\underbrace{\{1,1,1, \cdots, 1\}}_{b} .
$$

(c) Neutron. Neutron is a composite particle represented by $\mathbb{N}=\{0,1\}$. The mass of neutron is $M_{p}+M_{e}$. The set of $c$ neutrons is represented as

$$
\mathbb{N}^{c}=\underbrace{\{\{0,1\},\{0,1\},\{0,1\}, \cdots,\{0,1\}\}}_{c} .
$$

(d) Atomic nucleus. An atomic nucleus contains $b$ protons and $c$ neutrons,

$$
\mathbb{N u n c} \| \mathbb{e u s}(b, c)=\mathbb{P}^{b} \cup \mathbb{N}^{c}=\left\{\mathbb{P}^{b}, \mathbb{N}^{c}\right\} \text {. }
$$

Atom nucleus contains primary particles $b+2 c$ and has mass $(b+c) M_{p}+c M_{e}$.

(e) Ion. An ion is made up of a nucleus and the electrons outside the nucleus, which is represented as

$$
\mathbb{I}^{Z_{I}}(a, b, c)=\mathbb{E}^{a} \cup \mathbb{N} \text { undeus }(b, c)=\left\{\mathbb{E}^{a},\left\{\mathbb{P}^{b}, \mathbb{N}^{c}\right\}\right\},
$$

where $a$ is the number of electrons outside the nucleus, $b$ is the number of protons (atomic number) and $c$ is the number of neutrons in the nucleus. $Z_{I}=b-a$ is the number of ionic charges. An ion contains primary particles $N_{I}=a+b+2 c$ and has mass $M_{I}=(b+c) M_{p}+(a+c) M_{e}$.

(f) Atom. Atom is a combination of the atomic core and the valence electrons, which is represented as

$$
\mathbb{A} \mathbb{A} \mathbb{M}(b, c)=\left\{\mathbb{E}^{a_{1}},\left\{\mathbb{E}^{a_{0}},\left\{\mathbb{P}^{b}, \mathbb{N}^{c}\right\}\right\}\right\},
$$

where $\mathbb{E}^{a_{1}}$ stands for valence electrons. $a_{0}+a_{1}=b$ is atomic number, and $c$ stands for different isotopes. For example,

$$
\mathbb{O}(c)=\left\{\mathbb{E}^{6},\left\{\mathbb{E}^{2},\left\{\mathbb{P}^{8}, \mathbb{N}^{c}\right\}\right\}\right\}
$$

represents the atom of oxygen. Three stable isotopes of oxygen are $\mathbb{O}(8), \mathbb{O}(9)$ and $\mathbb{O}(10)$, corresponding to oxygen-16, oxygen-17 and oxygen-18. An atom contains primary particles $N_{A}=2(b+c)$ and has mass 
$M_{A}=(b+c)\left(M_{p}+M_{e}\right)$.

(g) Molecule. A molecule is a composite particle made up of ions. If the molecule is made up of $m$ ions, then the molecule $\mathbb{M}^{Z_{M}}$ is represented as

$$
\mathbb{M}^{Z_{M}}=\left\{\mathbb{I}_{1}^{Z_{I 1}}, \mathbb{I}_{2}^{Z_{I 2}}, \cdots, \mathbb{I}_{k}^{Z_{I k}}, \cdots, \mathbb{I}_{m}^{Z_{I m}}\right\},
$$

where $Z_{M}=\sum_{k=1}^{m} Z_{I k}$ is the charge number of the molecule. A molecule contains primary particles $N_{M}=\sum_{k=1}^{m} N_{I k}$ and has mass $M_{M}=\sum_{k=1}^{m} M_{I k}$.

(h) Molecular cluster. The upper layer structure made up of molecules is called molecular cluster. If the molecular cluster consists of $n$ molecules, then the molecular cluster $\mathbb{C}^{Z_{C}}$ is a set of molecules,

$$
\mathbb{C}^{Z_{C}}=\left\{\mathbb{M}_{1}^{Z_{M 1}}, \mathbb{M}_{2}^{Z_{M 2}}, \cdots, \mathbb{M}_{k}^{Z_{M k}}, \cdots, \mathbb{M}_{n}^{Z_{M n}}\right\},
$$

where $Z_{C}=\sum_{k=1}^{n} Z_{M k}$ is the charge number of molecular cluster. A molecular cluster contains primary particles $N_{C}=\sum_{k=1}^{n} N_{M k}$ and has mass $M_{C}=\sum_{k=1}^{n} M_{M k}$.

More complex particles are described in a similar way. According to above reasoning, each particle has definite mass and finite amount of primary particles. All objects are composed of electrons and protons with nesting structures. The nesting structure of an object can be imagined as "ball within ball" or "wheel within wheel".

\subsection{Model of Structure}

For a specific kind of substance, an object usually contains many layers of particles. For example, the water may include the cluster of water molecules, the molecule of water, the atoms of hydrogen and oxygen, and the primary particles. In order to study the properties of arbitrary object, it is necessary to establish a structure model of simple and universal significance.

When studying the properties of water, it usually involves the layers near molecule, and does not necessarily consider the layers below the atom. Generally speaking, the property of an object is only affected by particles in adjacent layers. This fact is called the effect of layer shield. According to the layer shield effect, an object model of four-layer particles is sufficient for general research.

An object under study is called a top-particle (top-layer), which is regarded as a system composed of $N$ base-particles (base-layer). In terms of set theory, an object is a finite countable set of $N$ base-particles. If $P_{i}$ represents the $i$-th base-particle, the object can be represented by a set of particles

$$
\text { Object }=\left\{P_{i} \mid i=1,2,3, \cdots, N\right\}=\left\{P_{i}\right\} \text {. }
$$

$\left\{P_{i}\right\}$ is a simplified form of the particle system in the convention of $i=1,2,3, \cdots, N$. Let $M_{i}$ be the mass of the $i$-th base-particle, then the mass of the object can be written as

$$
M=\sum_{i=1}^{N} M_{i}=N \cdot \sum_{i=1}^{N}\left(\frac{M_{i}}{N}\right) \equiv N \cdot \bar{M}, \quad \bar{M} \equiv \sum_{i=1}^{N}\left(\frac{M_{i}}{N}\right),
$$

where $\bar{M}$ is the average mass of base-particles. The symbol " $\equiv$ " is used as both identity sign and definition sign in this article.

The particles under the layer of base-particle are called sub-particles (sub-layer). The particles between the layers of top-particle and base-particle are called meso-particles (meso-layer). "Top-particle $\supseteq$ meso-particle $\supseteq$ base-particle $\supseteq$ sub-particle" forms a nesting structure of an object, as sketched in Fig. 1 . It should be stressed that these four layers are not independent of each other, but a nested structure that an upper layer comprises all lower layers.

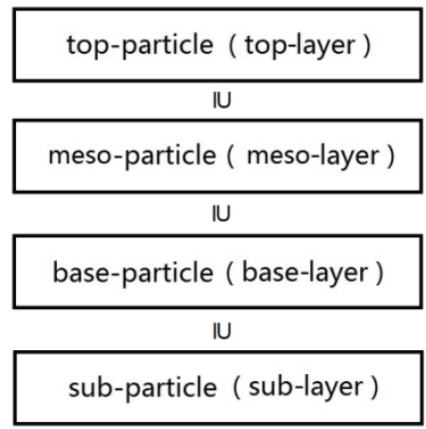

Figure 1. Model of object structure 
The base-particle is a relative concept, and sub-particle and meso-particle both are relative to the base-particle. Atom, molecule, molecular cluster, etc. can all be regarded as base-particles. For example, if the molecules are regarded as base-particles, then the atoms are sub-particles and the molecular clusters are meso-particles. In particle field theory, if the electronic field is regarded as an object, then the electrons are base-particles and the gridons are meso-particles [5]. The layers of the object can be selected according to the specific situation, not every layer must be considered.

Generally speaking, the top-particle is the object under study, and the base-particles are statistical elements. It is mathematically required that the number of base-particles $N$ is a system constant. For a general system, $N$ should be large enough to meet the law of large numbers in statistics. Physically, base-particles are required to be separate and elastic. Separation is the premise of countability. Elasticity ensures a stable structure in the collision of particles. Elastic deformation will lead to particle vibration. Therefore, elastic vibration is one of the important properties of the base-particles.

\subsection{Position of Particles}

The position of the basic-particle is represented by the position vector of the center of mass. In a laboratory frame of reference $O-X Y Z$, if the $i$-th particle has the mass $M_{i}$, and the center of mass is $P_{i}$, the line segment connecting the origin $O$ with $P_{i}$ is the position vector of the particle denoted by

where $x_{i}, y_{i}, z_{i}$ are Cartesian components of $\boldsymbol{r}_{i}$.

$$
\boldsymbol{r}_{i} \equiv \overrightarrow{O P_{l}}=\left(x_{i}, y_{i}, z_{i}\right)
$$

The time is defined by $[5]$

$$
t \equiv t_{s} \cdot \tilde{t} ; \quad \tilde{t}=0,1,2, \cdots, k, \cdots, N_{t} .
$$

where $t_{s}$ is the scale of time and $\tilde{t}$ is the digit of time. $\tilde{t}=0$ is the starting time, $\tilde{t}=N_{t}$ is the ending time, and $\tilde{t}=k$ is any time. The position $P_{i}$ at different time are expressed by an ordered time series

$$
\mathbb{r}_{i}(\tilde{t})=\left\langle\boldsymbol{r}_{i}(0), \boldsymbol{r}_{i}(1), \cdots, \boldsymbol{r}_{i}(k), \cdots, \boldsymbol{r}_{i}\left(N_{t}\right)\right\rangle \text {. }
$$

By convention, set elements are put in the braces \{\} , and ordered elements put in the brackets \langle\rangle . The set of positions $\mathbb{r}_{i}(\tilde{t})$ is the discrete representation of the continuous motion $\mathbb{r}_{i}(t)$ with the cardinality of $N_{t}+1$. The set of all particle positions is $\left\{\mathrm{r}_{i}(\tilde{t})\right\}$ with the cardinality of $N\left(N_{t}+1\right)$.

\subsection{Spatial State of Object}

An object is an entity having both mass and volume. The state of an object in three-dimensional space includes position, posture and profile. The position indicates the spatial location, the posture indicates the spatial orientation, and the profile indicates the outline of the object. The spatial state of an object is sketched in Fig. 2.

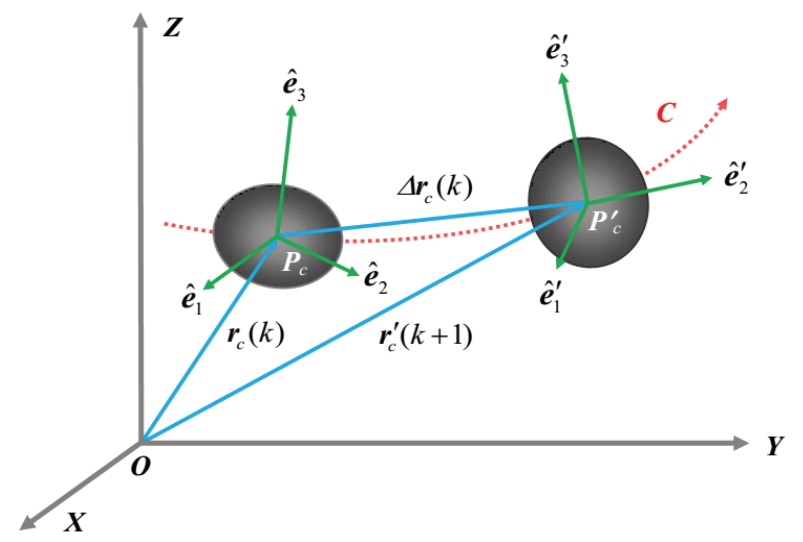

Figure 2. The spatial state of object: position, posture and profile.

The spatial state of an object can be determined by the configuration of base-particle system. The position of an object is specified as its center of mass in a laboratory frame of reference $(O-X Y Z)$. The 
center of mass $P_{c}$ is calculated as

$$
\boldsymbol{r}_{c}=\overrightarrow{O P_{c}}=\left(x_{c}, y_{c}, z_{c}\right)=\sum_{i=1}^{N}\left(\frac{M_{i}}{M}\right) \boldsymbol{r}_{i} .
$$

A coordinate frame with $P_{c}$ as the origin is called center-of-mass frame.

Posture and profile are determined by the inertia matrix of the object. The inertia matrix of the object in the laboratory frame is [1]

where

$$
\boldsymbol{I}=\left(\begin{array}{ccc}
I_{11} & -I_{12} & -I_{13} \\
-I_{21} & I_{22} & -I_{23} \\
-I_{31} & -I_{32} & I_{33}
\end{array}\right), \quad I_{s}=M_{s} r_{S}^{2}
$$

$$
\begin{aligned}
& I_{11}=\sum_{i=1}^{N} M_{i}\left(y_{i}^{2}+z_{i}^{2}\right), \quad I_{22}=\sum_{i=1}^{N} M_{i}\left(z_{i}^{2}+x_{i}^{2}\right), \quad I_{33}=\sum_{i=1}^{N} M_{i}\left(x_{i}^{2}+y_{i}^{2}\right), \\
& I_{12}=I_{21}=\sum_{i=1}^{N} M_{i} x_{i} y_{i}, \quad I_{31}=I_{13}=\sum_{i=1}^{N} M_{i} z_{i} x_{i}, \quad I_{23}=I_{32}=\sum_{i=1}^{N} M_{i} y_{i} z_{i} .
\end{aligned}
$$

The inertia matrix has nine components, but only six are independent because of its symmetry. The diagonal components $\left(I_{11}, I_{22}, I_{33}\right)$ are the rotary inertias about the coordinate axes $(X, Y, Z)$, and the non-diagonal components $\left(I_{12}, I_{23}, I_{31}\right)$ are the inertia products.

The inertia matrix is a real symmetric matrix and can be diagonalized in the center-of-mass frame [6]. After the diagonalization, the inertia products are all zero, leaving only the diagonal components $I_{1}, I_{2}, I_{3}$ are not zero. The coordinate axes that make the inertia products all zero are the principal axes of the object. The unit vectors in the directions of the principal axis are $\left(\hat{\mathbf{e}}_{1}, \hat{\mathbf{e}}_{2}, \hat{\mathbf{e}}_{3}\right)$. The included angles between $\left(\hat{\mathbf{e}}_{1}, \hat{\mathbf{e}}_{2}, \hat{\mathbf{e}}_{3}\right)$ and $(X, Y, Z)$ are marked as $\boldsymbol{\theta}_{c}=\left(\theta_{1}, \theta_{2}, \theta_{3}\right)$, which is adopted to characterize the posture of the object.

The non-zero diagonal components $I_{1}, I_{2}, I_{3}$ are the principal rotary inertias about the three principal axes. According to the theory of linear algebra [6], the principal rotary inertias $I_{1}, I_{2}, I_{3}$ are the eigenvalues of the inertia matrix. The principal axes $\hat{\mathbf{e}}_{1}, \hat{\mathbf{e}}_{2}, \hat{\mathbf{e}}_{3}$ are the normalized eigenvectors corresponding to the three eigenvalues. For convenience, the principal rotary inertias are marked as $\boldsymbol{I}_{c}=\left(I_{1}, I_{2}, I_{3}\right)$, which is adopted to characterize the profile of the object. The profile of the object may be conceived as an ellipsoid defined with the principal rotary inertias

$$
\left(\frac{x}{a}\right)^{2}+\left(\frac{x}{b}\right)^{2}+\left(\frac{x}{c}\right)^{2}=1 ; a=\sqrt{I_{1} / M}, b=\sqrt{I_{2} / M}, c=\sqrt{I_{3} / M} .
$$

\section{Motion and Energy}

\subsection{Modes of Motion}

Motion is a phenomenon that an object changes its spatial state with time in space. As showed in Fig. 2 , the position of an object at any time $\tilde{t}=k$ is at $P_{c}$ with the position vector $\boldsymbol{r}_{c}=\left(x_{c}, y_{c}, z_{c}\right)$. The corresponding posture and profile are $\boldsymbol{\theta}_{c}=\left(\theta_{1}, \theta_{2}, \theta_{3}\right)$ and $\boldsymbol{I}_{c}=\left(I_{1}, I_{2}, I_{3}\right)$. At the next time $\tilde{t}=k+1$, the position shifts to $P^{\prime}{ }_{c}$ with the position vector $\boldsymbol{r}_{c}^{\prime}=\left(x^{\prime}, y_{c}^{\prime}, z_{c}^{\prime}\right)$. The corresponding posture and profile becomes $\boldsymbol{\theta}_{c}^{\prime}=\left(\theta_{1}^{\prime}, \theta_{2}^{\prime}, \theta^{\prime}{ }_{3}\right)$ and $\boldsymbol{I}_{c}^{\prime}=\left(I_{1}^{\prime}, I^{\prime}{ }_{2}, I^{\prime}{ }_{3}\right)$. Then, the change of spatial states is expressed as

$$
\begin{gathered}
\Delta \boldsymbol{r}_{c}=\boldsymbol{r}^{\prime}{ }_{c}-\boldsymbol{r}_{c}=\left(\Delta x_{c}, \Delta y_{c}, \Delta z_{c}\right), \\
\Delta \boldsymbol{\theta}_{c}=\boldsymbol{\theta}^{\prime}{ }_{c}-\boldsymbol{\theta}_{c}=\left(\Delta \theta_{1}, \Delta \theta_{2}, \Delta \theta_{3}\right), \\
\Delta \boldsymbol{I}_{c}=\boldsymbol{I}^{\prime}{ }_{c}-\boldsymbol{I}_{c}=\left(\Delta I_{1}, \Delta I_{2}, \Delta I_{3}\right) .
\end{gathered}
$$

The change of position $\Delta \boldsymbol{r}_{c}$ represents a translation, the change of posture $\boldsymbol{\Delta} \boldsymbol{\theta}_{c}$ represents a rotation, and the change of profile $\Delta \boldsymbol{I}_{c}$ represents a vibration. Translation is the displacement of the center-of-mass. Rotation is the angle change around the center of mass. The vibration is caused by the deformation of profile of the elastic object. In the center-of-mass frame, the rotation is a pure angular change and the vibration is a pure radial change. Therefore, the translation, rotation and vibration are three independent modes of motion. 
According to the structure model, an object is a system containing $N$ base-particles. Each particle has three independent modes of motion, and each mode has three independent components. Therefore, an object contains $9 \mathrm{~N}$ internal degrees of freedom of motion.

\subsection{Translating Motion}

\subsubsection{Translating velocity}

The position of a base-particle at the time $\tilde{t}=k$ is $\boldsymbol{r}_{i}=\left(x_{i}, y_{i}, z_{i}\right)$, and at next time $\tilde{t}=k+1$ is $\boldsymbol{r}_{i}^{\prime}=\left(x_{i}^{\prime}, y_{i}^{\prime}, z_{i}^{\prime}\right)$. The translation can be expressed as

$$
\Delta \boldsymbol{r}_{i}(k)=\overrightarrow{P_{l} P^{\prime}}=\left(\Delta x_{i}, \Delta y_{i}, \Delta z_{i}\right)=r_{s} \cdot \Delta \tilde{\boldsymbol{r}}_{i}(k) ; \Delta \tilde{\boldsymbol{r}}_{i}(k)=\left(\Delta \tilde{x}_{i}, \Delta \tilde{y}_{i}, \Delta \tilde{z}_{i}\right)
$$

The translating velocity is defined as

$$
\begin{gathered}
\boldsymbol{u}_{i}(k) \equiv \Delta \boldsymbol{r}_{i}(k) / t_{s}=\left(r_{s} / t_{s}\right) \cdot \Delta \tilde{\boldsymbol{r}}_{i}(k)=u_{s} \cdot \tilde{\boldsymbol{u}}_{i}(k) ; \\
u_{s}=r_{s} / t_{s}, \quad \tilde{\boldsymbol{u}}_{i}(k)=\Delta \tilde{\boldsymbol{r}}_{i}(k)=\left(\tilde{u}_{i 1}, \tilde{u}_{i 2}, \tilde{u}_{i 3}\right) .
\end{gathered}
$$

$\boldsymbol{u}_{i}(k)$ are origin irrelevant since they are independent of the laboratory frame of reference.

\subsubsection{Translating energy}

The translating momentum of the particle is defined as

$$
\boldsymbol{p}_{i} \equiv M_{i} \boldsymbol{u}_{i}=\left(p_{i 1}, p_{i 2}, p_{i 3}\right)=p_{s} \cdot \widetilde{\boldsymbol{p}}_{i} ; \quad p_{s}=M_{s} u_{s}, p_{i \alpha}=M_{i} u_{i \alpha}, \alpha=1,2,3 .
$$

The translating energy of the particle is defined as

$$
K_{i} \equiv \frac{1}{2} \boldsymbol{p}_{i} \cdot \boldsymbol{u}_{i}=\frac{1}{2} M_{i}\left(\boldsymbol{u}_{i} \cdot \boldsymbol{u}_{i}\right)=\sum_{\alpha=1}^{3} K_{i \alpha} ; K_{i \alpha}=\frac{p_{i \alpha}^{2}}{2 M_{i}}, K_{s}=p_{s} u_{s}=M_{s} u_{s}^{2},
$$

where $K_{s}$ is the scale of translating energy. It is seen that $\boldsymbol{u}_{i}$ and $\boldsymbol{p}_{i}$ each has three components, and $K_{i}$ has three independent square terms, which indicates that each particle has 3 degrees of freedom of translation.

The total translating energy $K$ is obtained by summing all particles. It contains $3 N$ independent square terms.

$$
\begin{aligned}
K & =\sum_{i=1}^{N} K_{i}=\sum_{i=1}^{N}\left(\sum_{\alpha=1}^{3} K_{i \alpha}\right)=\sum_{\alpha=1}^{3}\left(\sum_{i=1}^{N} K_{i \alpha}\right) \\
& =\sum_{\alpha=1}^{3} K_{\alpha}, \quad K_{\alpha}=\sum_{i=1}^{N} K_{i \alpha} .
\end{aligned}
$$

\subsubsection{Scale of translating energy}

We define the scale of translating energy as follows

$$
K_{s} \equiv K / N=K / \widetilde{K} ; \widetilde{K}=N \text {. }
$$

It means that the digit of total translating energy is equal to the number of particles.

Choosing a translating constant $k$ for scale transformation, the scale of translating energy $K_{s}$ can be replaced by the translating intensity $T$.

$$
K_{s}=k\left(K_{s} / k\right) \equiv k T ; \quad T \equiv K_{s} / k=K /(N k) .
$$

The translating intensity $T$ is exactly the thermodynamic temperature. In SI system, the energy unit is joule $(\mathrm{J})$ and the temperature unit is kelvin $(\mathrm{K})$, then the translating constant is just the Boltzmann constant $k=1.3806506 \times 10^{-23} \mathrm{~J} \cdot \mathrm{K}^{-1}$.

Let $K_{s}$ in formulas $(24)$ and (27) be equal, we get following scale relation for the translating energy

$$
K_{s}=M_{s} u_{s}^{2}=k T \text {. }
$$

\subsection{Rotating Motion}

\subsubsection{Rotating velocity}

The posture of a base-particle at the time $\tilde{t}=k$ is $\boldsymbol{\theta}_{i}=\left(\theta_{i 1}, \theta_{i 2}, \theta_{i 3}\right)$, and at next time $\tilde{t}=k+1$ is $\boldsymbol{\theta}_{i}^{\prime}=\left(\theta_{i 1}^{\prime}, \theta^{\prime}{ }_{i 2}, \theta^{\prime}{ }_{i 3}\right)$. The rotation can be expressed as

$$
\Delta \boldsymbol{\theta}_{i}(k)=\left(\Delta \theta_{i 1}, \Delta \theta_{i 2}, \Delta \theta_{i 3}\right)=\theta_{s} \cdot \Delta \widetilde{\boldsymbol{\theta}}_{i}(k) ;
$$


The rotating velocity is defined as

$$
\theta_{s}=1, \Delta \widetilde{\boldsymbol{\theta}}_{i}(k)=\left(\Delta \tilde{\theta}_{i 1}, \Delta \tilde{\theta}_{i 2}, \Delta \tilde{\theta}_{i 3}\right) .
$$

$$
\begin{gathered}
\boldsymbol{\omega}_{i}(k) \equiv \Delta \boldsymbol{\theta}_{i}(k) / t_{s}=\left(\theta_{s} / t_{s}\right) \cdot \Delta \widetilde{\boldsymbol{\theta}}_{i}(k)=\omega_{s} \cdot \widetilde{\boldsymbol{\omega}}_{i}(k)=\left(\omega_{i 1}, \omega_{i 2}, \omega_{i 3}\right) ; \\
\omega_{s}=1 / t_{s}, \quad \widetilde{\boldsymbol{\omega}}_{i}(k)=\Delta \widetilde{\boldsymbol{\theta}}_{i}(k)=\left(\Delta \tilde{\theta}_{i 1}, \Delta \tilde{\theta}_{i 2}, \Delta \tilde{\theta}_{i 3}\right) .
\end{gathered}
$$

The rotating velocity is also origin irrelevant.

\subsubsection{Rotating energy}

$I_{i 1}, I_{i 2}, I_{i 3}$ are three principal rotary inertias of a base-particle at the time $\tilde{t}=k$, the corresponding inertia matrix is

$$
\boldsymbol{I}_{i}=\left(\begin{array}{ccc}
I_{i 1} & 0 & 0 \\
0 & I_{i 2} & 0 \\
0 & 0 & I_{i 3}
\end{array}\right) .
$$

Then, the rotating momentum of the particle is defined as

$$
\begin{gathered}
\boldsymbol{s}_{i} \equiv \boldsymbol{I}_{i} \cdot \boldsymbol{\omega}_{i}=s_{s} \cdot \tilde{\boldsymbol{s}}_{i}=\left(s_{i 1}, s_{i 2}, s_{i 3}\right) ; \\
s_{s}=I_{s} \omega_{s}, \quad s_{i \alpha}=I_{i \alpha} \omega_{i \alpha}, \alpha=1,2,3 .
\end{gathered}
$$

The rotating energy of the particle is defined as

$$
\begin{gathered}
L_{i} \equiv \frac{1}{2} \boldsymbol{\omega}_{i}^{T} \cdot \boldsymbol{I}_{i} \cdot \boldsymbol{\omega}_{i}=\frac{1}{2} \boldsymbol{\omega}_{i}^{T} \cdot \boldsymbol{s}_{i}=\sum_{\alpha=1}^{3} L_{i \alpha} ; \\
L_{i \alpha}=\frac{s_{i \alpha}^{2}}{2 I_{i \alpha}}, \quad L_{s}=\omega_{s} s_{s}=I_{s} \omega_{s}^{2} .
\end{gathered}
$$

where $\boldsymbol{\omega}_{i}^{T}$ is the transposition of $\boldsymbol{\omega}_{i}$, and $L_{s}$ is the scale of rotating energy. It is seen that $\boldsymbol{\omega}_{i}$ and $\boldsymbol{s}_{i}$ each has three components, and $L_{i}$ has three independent square terms, which indicates that each particle has 3 degrees of freedom of rotation.

The total rotating energy $L$ is obtained by summing all particles. It contains $3 N$ independent square terms.

$$
\begin{aligned}
L & =\sum_{i=1}^{N} L_{i}=\sum_{i=1}^{N}\left(\sum_{\alpha=1}^{3} L_{i \alpha}\right)=\sum_{\alpha=1}^{3}\left(\sum_{n=1}^{N} L_{i \alpha}\right) \\
& =\sum_{\alpha=1}^{3} L_{\alpha}, \quad L_{\alpha}=\sum_{n=1}^{N} L_{i \alpha} .
\end{aligned}
$$

\subsubsection{Scale of rotating energy}

The scale of rotating energy is defined as follows

$$
L_{s} \equiv L / N=L / \tilde{L} ; \tilde{L}=N \text {. }
$$

It means that the digit of total rotating energy is equal to the number of particles.

Choosing a rotating constant $l$ for scale transformation, the scale of rotating energy $L_{s}$ can be replaced by the rotating intensity $z$.

$$
L_{s}=l\left(L_{s} / l\right) \equiv l z ; \quad z \equiv L_{s} / l=L /(N l) .
$$

Let $L_{s}$ in formulas (33) and (36) be equal, we get following scale relation for the rotating energy.

$$
L_{s}=I_{s} \omega_{s}^{2}=l z \text {. }
$$

\subsection{Vibrating Motion}

\subsubsection{Particle deformation}

The principal rotary inertia at the time $\tilde{t}=k$ is $\boldsymbol{I}_{i c}=\left(I_{i 1}, I_{i 2}, I_{i 3}\right)$, and at next time $\tilde{t}=k+1$ is $\boldsymbol{I}^{\prime}{ }_{i c}=\left(I^{\prime}{ }_{i 1}, I^{\prime}{ }_{i 2}, I^{\prime}{ }_{i 3}\right)$. The deformation of the particle is defined as

$$
\begin{aligned}
& \boldsymbol{\varepsilon}_{i}(k) \equiv\left(\boldsymbol{I}^{\prime}{ }_{i c}-\boldsymbol{I}_{i c}\right) / I_{i}=\left(\varepsilon_{i 1}, \varepsilon_{i 2}, \varepsilon_{i 3}\right), \quad \varepsilon_{s}=1, \\
& I_{i}=I_{i 1}+I_{i 2}+I_{i 3}, \quad \varepsilon_{i \alpha}=\Delta I_{i \alpha} / I_{i}, \quad \alpha=1,2,3 .
\end{aligned}
$$

The deformation of a particle has three components. 


\subsubsection{Vibrating energy}

If the principal elastic modulus of the particle are $Y_{i 1}, Y_{i 2}, Y_{i 3}$ corresponding to the deformation $\varepsilon_{i 1}, \varepsilon_{i 2}, \varepsilon_{i 3}$, then the energy density of the elastic deformation can be expressed as [7]

$$
F_{i}=\sum_{\alpha=1}^{3}\left(\frac{1}{2} Y_{i \alpha} \varepsilon_{i \alpha}^{2}\right)=\sum_{\alpha=1}^{3}\left(\frac{\chi_{i \alpha}^{2}}{2 Y_{i \alpha}}\right) ; \quad F_{s}=Y_{S} \varepsilon_{s}^{2}=Y_{S},
$$

where $\chi_{i \alpha}$ is the component of principal stress, which is defined as

$$
\chi_{i \alpha} \equiv \frac{\partial F_{i}}{\partial \varepsilon_{i \alpha}}=Y_{i \alpha} \varepsilon_{i \alpha} ; \quad \chi_{s}=\frac{F_{s}}{\varepsilon_{s}}=Y_{s} .
$$

Let the volume of the particle be $V_{i}$, then the vibrating energy (the energy of elastic deformation) of the particle is

$$
H_{i}=V_{i} \cdot F_{i}=V_{i} \cdot \sum_{\alpha=1}^{3}\left(\frac{\chi_{i \alpha}^{2}}{2 Y_{i \alpha}}\right) ; \quad H_{s}=V_{S} F_{s}=V_{s} Y_{S} .
$$

The total vibrating energy $H$ of the object is obtained by summing all base-particles. It contains $3 \mathrm{~N}$ independent square terms.

$$
\begin{aligned}
H & =\sum_{i=1}^{N} H_{i}=\sum_{i=1}^{N}\left[V_{i} \sum_{\alpha=1}^{3}\left(\frac{\chi_{i \alpha}^{2}}{2 Y_{i \alpha}}\right)\right]=\sum_{\alpha=1}^{3}\left[\sum_{n=1}^{N}\left(\frac{V_{i} \chi_{i \alpha}^{2}}{2 Y_{i \alpha}}\right)\right] \\
& =\sum_{\alpha=1}^{3} H_{\alpha}, \quad H_{\alpha}=\sum_{n=1}^{N}\left(\frac{V_{i} \chi_{i \alpha}^{2}}{2 Y_{i \alpha}}\right) .
\end{aligned}
$$

\subsubsection{Scale of vibrating energy}

The scale of vibrating energy is defined as follows

$$
H_{s} \equiv H / N=H / \widetilde{H} ; \widetilde{H}=N .
$$

It means that the digit of total vibrating energy is equal to the number of particles.

Choosing a vibrating constant $h$ for scale transformation, the scale of vibrating energy $H_{s}$ can be replaced by the vibrating intensity $v$.

$$
H_{s}=h\left(H_{s} / h\right) \equiv h v ; \quad v \equiv H_{s} / h=H /(N h) .
$$

The vibrating intensity $v$ is exactly the frequency. In SI system, the unit of frequency takes hertz $(\mathrm{Hz})$, then the vibrating constant is just the Planck constant $h=6.6260690 \times 10^{-34} \mathrm{~J} \cdot \mathrm{Hz}^{-1}$.

Let $H_{S}$ in formulas (41) and (44) be equal, we get following scale relation for the vibrating energy.

$$
H_{s}=V_{s} Y_{S}=h v \text {. }
$$

\section{$5 \quad$ Property of Energy Space}

\subsection{Motion Persistence}

In the center-of-mass frame, the motion of a body particle can be decomposed into three independent modes: translation, rotation and vibration. Each mode has 3 degrees of freedom, each particle has 9 degrees of freedom, and $N$ particles have a total of $9 N$ independent energy terms. The total energies of vibration, rotation and translation are given as follows.

$$
\begin{aligned}
H & =H_{s} \cdot \widetilde{H}=\sum_{i=1}^{N} H_{i}=\sum_{\alpha=1}^{3} H_{\alpha}, \\
L & =L_{s} \cdot \widetilde{L}=\sum_{i=1}^{N} L_{i}=\sum_{\alpha=1}^{3} L_{\alpha}, \\
K & =K_{s} \cdot \widetilde{K}=\sum_{i=1}^{N} K_{i}=\sum_{\alpha=1}^{3} K_{\alpha} .
\end{aligned}
$$

$\{H, L, K\}$ is collectively called the motion energy of the object. $H, L$ and $K$ are three components of the motion energy.

Since each component is comprised of independent square terms, the motion energy is non-zero and always positive. 


$$
H>0, \quad L>0, \quad K>0 .
$$

The positive definiteness of motion energy is called the theorem of motion persistence.

Motion persistence means that particles inside an object are in a state of motion that never stops. That's the reason why objects have volume. $K=N k T>0$, namely $T>0$, is the statement of the third law of thermodynamics. $H=N h v>0$, namely $v>0$, is the zero-point energy assumed by quantum mechanics.

\subsection{Energy Space}

For research purposes, we make an order convention for the total energies of the three modes of motion

$$
E_{1}=H, \quad E_{2}=L, \quad E_{3}=K .
$$

Now, we use the components of motion energy as the set elements to form three ordered zones

$$
\begin{gathered}
\mathbb{E}^{h} \equiv\left\langle E_{2}^{h}, E_{3}^{h}, E_{1}^{h}\right\rangle=\left\langle L^{h}, K^{h}, H^{h}\right\rangle, \\
\mathbb{E}^{l} \equiv\left\langle E_{3}^{l}, E_{1}^{l}, E_{2}^{l}\right\rangle=\left\langle K^{l}, H^{l}, L^{l}\right\rangle, \\
\mathbb{E}^{k} \equiv\left\langle E_{1}^{k}, E_{2}^{k}, E_{3}^{k}\right\rangle=\left\langle H^{k}, L^{k}, K^{k}\right\rangle .
\end{gathered}
$$

The mathematical space formed by sets $\mathbb{E}^{h}, \mathbb{E}^{l}, \mathbb{E}^{k}$ is called the energy space of objects. $\mathbb{E}^{h}, \mathbb{E}^{l}, \mathbb{E}^{k}$ are respectively referred to as gas zone, solid zone, and liquid zone (The reason will be explained later). The superscript $x=h, l, k$ is the zone index (not the power index). Under the order convention, $\left\{L^{h}, K^{l}, H^{k}\right\}$ is called the ahead energy, $\left\{K^{h}, H^{l}, L^{k}\right\}$ is called the back energy, and $\left\{H^{h}, L^{l}, K^{k}\right\}$ is called the major energy.

It can be seen from the definition (49) that the three ordered zones have permutation symmetry. By the even permutation of $E_{1}, E_{2}, E_{3}$, any zone can be transformed into the other two zones. That is, the energy space has the zone symmetry under even permutation of $E_{1}, E_{2}, E_{3}$. Using this property, the conclusion in one zone can be extended to other zones.

\subsection{Auxiliary Energy}

Four kinds of auxiliary energies are defined in the energy space as follows.

(1) Entire energy. $\left\{E^{h}, E^{l}, E^{k}\right\}$ is the sum of ahead energy and back energy.

$$
\begin{gathered}
E^{h} \equiv E_{2}^{h}+E_{3}^{h}=L^{h}+K^{h}, \\
E^{l} \equiv E_{3}^{l}+E_{1}^{l}=K^{l}+H^{l}, \\
E^{k} \equiv E_{1}^{k}+E_{2}^{k}=H^{k}+L^{k} .
\end{gathered}
$$

(2) Thermal energy. $\left\{Q^{h}, Q^{l}, Q^{k}\right\}$ is the sum of back energy and major energy.

$$
\begin{gathered}
Q^{h} \equiv E_{3}^{h}+E_{1}^{h}=K^{h}+H^{h}, \\
Q^{l} \equiv E_{1}^{l}+E_{2}^{l}=H^{l}+L^{l}, \\
Q^{k} \equiv E_{2}^{k}+E_{3}^{k}=L^{k}+K^{k} .
\end{gathered}
$$

(3) Potential energy. $\left\{J^{h}, J^{l}, J\right\}$ is the difference between ahead energy and major energy.

$$
\begin{gathered}
J^{h} \equiv E_{2}^{h}-E_{1}^{h}=L^{h}-H^{h}, \\
J^{l} \equiv E_{3}^{l}-E_{2}^{l}=K^{l}-L^{l}, \\
J^{k} \equiv E_{1}^{k}-E_{3}^{k}=H^{k}-K^{k} .
\end{gathered}
$$

(4) Chemical energy. $\left\{G^{h}, G^{l}, G^{k}\right\}$ is the difference between back energy and ahead energy.

$$
\begin{aligned}
& G^{h} \equiv E_{3}^{h}-E_{2}^{h}=K^{h}-L^{h}, \\
& G^{l} \equiv E_{1}^{l}-E_{3}^{l}=H^{l}-K^{l}, \\
& G^{k} \equiv E_{2}^{k}-E_{1}^{k}=L^{k}-H^{k} .
\end{aligned}
$$

The auxiliary energy introduced here is the thermodynamic potential [8]. It is proved [4] that $J^{k}$ is the potential energy and $G^{k}$ is the Gibbs free energy. $Q^{k}$ is a physical quantity that replaces heat and does not depend on thermodynamic processes. In addition, $H^{k}$ is the mechanical energy (not the enthalpy), $-H^{k}$ is the Helmholtz free energy and $-L^{k}$ is the grand potential.

\subsection{State Vector}

Energy space needs to be geometrized in order to describe the structure visually and analyze the state change intuitively. A geometrized energy space $O-E_{1} E_{2} E_{3}$ is a 3-dimensional Cartesian space constructed with the components of motion energy. A state vector $\boldsymbol{E}^{x}$ is defined in $O-E_{1} E_{2} E_{3}$ as follows

$$
\boldsymbol{E}^{x} \equiv\left(E_{1}^{x}, E_{2}^{x}, E_{3}^{x}\right)=E_{1}^{x} \mathbf{i}+E_{2}^{x} \mathbf{j}+E_{3}^{x} \mathbf{k}=E^{x} \mathbf{e}_{0},
$$




$$
E^{x} \equiv \sqrt{\left(E_{1}^{x}\right)^{2}+\left(E_{2}^{x}\right)^{2}+\left(E_{3}^{x}\right)^{2}}=\sqrt{\left(H^{x}\right)^{2}+\left(L^{x}\right)^{2}+\left(K^{x}\right)^{2}},
$$

where $x=h, l, k$ is the zone index, $\mathbf{i}, \mathbf{j}, \mathbf{k}$ are the standard bases of Cartesian coordinate, $\mathbf{e}_{0}$ is the unit vector in the direction of the state vector, and $E^{x}$ is the length of the state vector. According to the positive definiteness of $E_{1}, E_{2}, E_{3}$, the energy space is confined to the first octant $(+,+,+)$ of the Cartesian space.

\subsection{Equilibrium State}

It is stipulated that the length of the state vector is equal to the entire energy defined by the auxiliary energy, namely, the following constraints are imposed on the energy space

$$
\begin{aligned}
& E^{h}=\sqrt{\left(E_{1}^{h}\right)^{2}+\left(E_{2}^{h}\right)^{2}+\left(E_{3}^{h}\right)^{2}}=E_{2}^{h}+E_{3}^{h}, \\
& E^{l}=\sqrt{\left(E_{1}^{l}\right)^{2}+\left(E_{2}^{l}\right)^{2}+\left(E_{3}^{l}\right)^{2}}=E_{3}^{l}+E_{1}^{l}, \\
& E^{k}=\sqrt{\left(E_{1}^{k}\right)^{2}+\left(E_{2}^{k}\right)^{2}+\left(E_{3}^{k}\right)^{2}}=E_{1}^{k}+E_{2}^{k} .
\end{aligned}
$$

It gives following conditions representing the equilibrium states of the object.

$$
\left(H^{h}\right)^{2}=2 L^{h} K^{h}, \quad\left(L^{l}\right)^{2}=2 K^{l} H^{l}, \quad\left(K^{k}\right)^{2}=2 H^{k} L^{l} .
$$

\subsection{Space Structure}

In order to describe the structure of energy space, 3-dimensional region (body) is marked with symbol $\mathrm{B}[*]$, 2-dimensional region (surface) is marked with symbol $\mathrm{S}[*]$, and 1-dimensional region (line) is marked with symbol $\mathrm{L}[*]$.
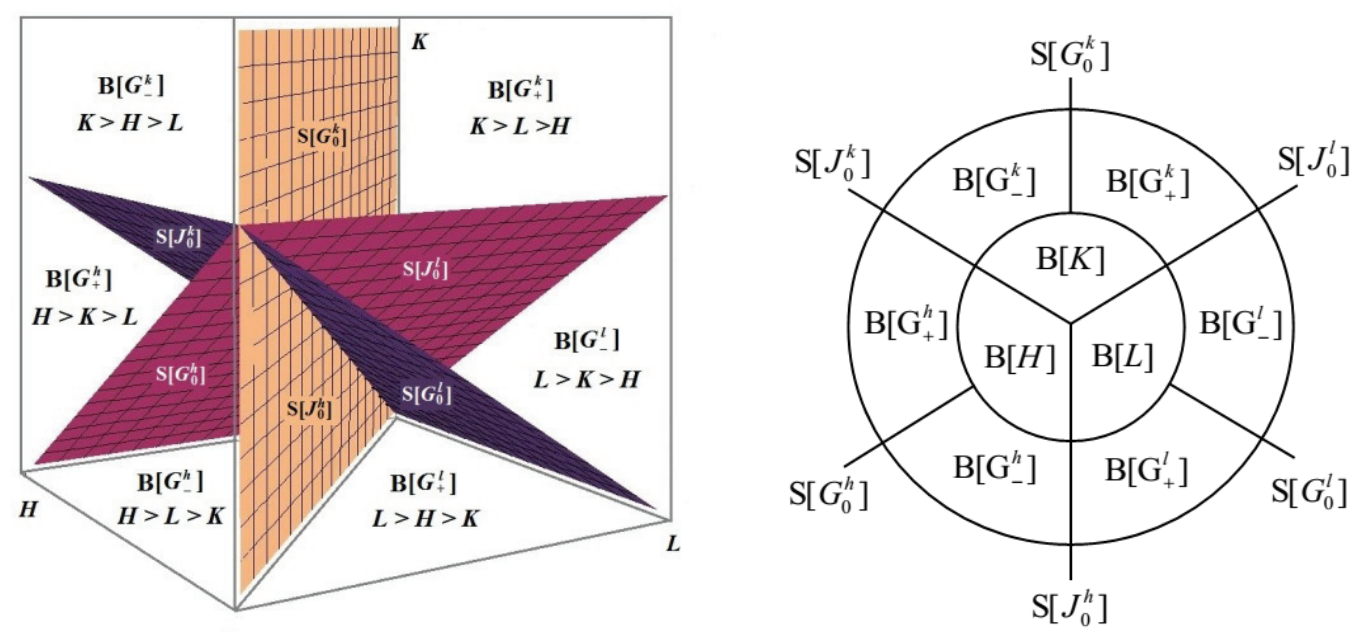

Figure 3. Structure of energy space. (a) Three-dimensional Cartesian space. (b) Two-dimensional mapping.

As shown in Fig. 3(a), The energy space contains coordinate axes $H, L, K$, and the boundary includes the coordinate planes $(\mathrm{S}[H=0], \mathrm{S}[L=0]$ and $\mathrm{S}[K=0])$. The energy space is divided by three planes $(\mathrm{S}[H=K], \mathrm{S}[K=L]$ and $\mathrm{S}[L=H])$ into six phases:
(1) Phase $\mathrm{B}\left[G_{+}^{h}\right]=\mathrm{B}\left[G^{h}=K^{h}-L^{h}>0\right]: H>K>L$;
(2) Phase $\mathrm{B}\left[G_{-}^{h}\right]=\mathrm{B}\left[G^{h}=K^{h}-L^{h}<0\right]: H>L>K$;
(3) Phase $\mathrm{B}\left[G_{+}^{l}\right]=\mathrm{B}\left[G^{l}=H^{l}-K^{l}>0\right]: L>H>K$;
(4) Phase $\mathrm{B}\left[G_{-}^{l}\right]=\mathrm{B}\left[G^{l}=H^{l}-K^{l}<0\right]: L>K>H$;
(5) Phase $\mathrm{B}\left[G_{+}^{k}\right]=\mathrm{B}\left[G^{k}=L^{k}-H^{k}>0\right]: K>L>H$;
(6) Phase $\mathrm{B}\left[G_{-}^{k}\right]=\mathrm{B}\left[G^{k}=L^{k}-H^{k}<0\right]: K>H>L$.

The six phases are grouped into three zones:

(1) Zone $\mathrm{B}[H]=\mathrm{B}\left[G_{+}^{h}\right]+\mathrm{B}\left[G_{-}^{h}\right]$; 
(2) Zone $\mathrm{B}[L]=\mathrm{B}\left[G_{+}^{l}\right]+\mathrm{B}\left[G_{-}^{l}\right]$;

(3) Zone $\mathrm{B}[K]=\mathrm{B}\left[G_{+}^{k}\right]+\mathrm{B}\left[G_{-}^{k}\right]$.

$\mathrm{B}[H], \mathrm{B}[L]$ and $\mathrm{B}[K]$ correspond to $\mathbb{E}^{h}, \mathbb{E}^{l}$ and $\mathbb{E}^{k}$ zones. Because they are dominated by vibrating energy, rotating energy and translating energy, we called them gas zone, solid zone and liquid zone, respectively.

The six phases have six interfaces, which are divided into two types:

(1) J-type interface includes the planes of zero potential energy

$$
\begin{aligned}
& \mathrm{S}\left[J_{0}^{h}\right]=\mathrm{S}\left[J^{h}=L^{h}-H^{h}=0\right] ; \\
& \left.\mathrm{S} J_{0}^{l}\right]=\mathrm{S}\left[J^{l}=K^{l}-L^{l}=0\right] ; \\
& \mathrm{S}\left[J_{0}^{k}\right]=\mathrm{S}\left[J^{k}=H^{k}-K^{k}=0\right] .
\end{aligned}
$$

(2) G-type interface includes the planes of zero chemical energy

$$
\begin{aligned}
& \mathrm{S}\left[G_{0}^{h}\right]=\mathrm{S}\left[G^{h}=K^{h}-L^{h}=0\right] ; \\
& \mathrm{S}\left[G_{0}^{l}\right]=\mathrm{S}\left[G^{l}=H^{l}-K^{l}=0\right] ; \\
& \mathrm{S}\left[G_{0}^{k}\right]=\mathrm{S}\left[G^{k}=L^{k}-H^{k}=0\right] .
\end{aligned}
$$

The two types of interfaces predict two types of phase transitions. J-type is the phase transition across two zones, and G-type is the phase transition within a zone. $\mathrm{S}\left[J_{0}^{h}\right], \mathrm{S}\left[J_{0}^{l}\right]$ and $\mathrm{S}\left[J_{0}^{k}\right]$ are gas-solid interface, solid-liquid interface and liquid-gas interface, respectively. $S\left[G_{0}^{h}\right], S\left[G_{0}^{l}\right]$ and $S\left[G_{0}^{k}\right]$ are forbidden planes which will be discussed elsewhere. Fig. 3(a) shows the structure of the energy space. Fig. 3(b) is a planar mapping of the energy space.

\subsection{Order Parameter}

On the planar mapping of the energy space, we stipulate that the counterclockwise is the direction of positive rotation. Accordingly, the starting plane of the gas zone is $\mathrm{S}\left[J_{0}^{k}\right]$ and the ending plane is $\mathrm{S}\left[J_{0}^{h}\right]$; the starting plane of the solid zone is $\mathrm{S}\left[J_{0}^{h}\right]$ and the ending plane is $\mathrm{S}\left[J_{0}^{l}\right]$; the starting plane of the liquid zone is $S\left[J_{0}^{l}\right]$ and the ending plane is $S\left[J_{0}^{k}\right]$. We take liquid as an example to illustrate the property of ordered zones.

In the liquid zone $\mathrm{B}[K], H^{k}$ is ahead energy, $L^{k}$ is back energy and $K^{k}$ is major energy. Along the direction of positive rotation, the ahead energy increases while the back energy decreases. At the same time, the major energy is always greater than the other two components. Hence, the potential energy $J^{k}=H^{k}-K^{k}$ is always negative, and the chemical energy $G^{k}=L^{k}-H^{k}$ can be either negative or positive.

Now, we define two order parameters in the liquid zone: the ahead parameter $a^{k}$ and the back parameter $b^{k}$.

$$
a^{k} \equiv H^{k} / K^{k}, \quad b^{k} \equiv L^{k} / K^{k} .
$$

Then the equilibrium condition $\left(K^{k}\right)^{2}=2 H^{k} L^{l}$ can be expressed in terms of the order parameter.

$$
2 a^{k} b^{k}=1 \text {. }
$$

On the starting plane $\mathrm{S}\left[J_{0}^{l}\right]$, it has $L^{k}=K^{k}$, the ahead parameter $a^{k}=1 / 2$ and the back parameter $b^{k}=1$. On the ending plane $\mathrm{S}\left[J_{0}^{k}\right]$, it has $H^{k}=K^{k}$, the ahead parameter $a^{k}=1$ and the back parameter $b^{k}=1 / 2$. Therefore, the ahead parameter is monotonically increasing from $1 / 2$ to 1 , and the back parameter is monotonically decreasing from 1 to $1 / 2$, within the liquid zone.

In real physics, order parameter is a very important quantity. The order parameter in the equilibrium state can be obtained by statistics of particle characteristics inside the object. According to the permutation symmetry, we can define and analyze the order parameters in other two zones. Table 1 listed the results of order parameters in three zones.

Table 1. Order parameters in three zones

\begin{tabular}{ccccc}
\hline Zone & Ahead parameter & Back parameter & Equilibrium condition & Range \\
\hline $\mathrm{B}[H]$ & $a^{h}=L^{h} / H^{h}$ & $b^{h}=K^{h} / H^{h}$ & $2 a^{h} b^{h}=1$ & $1 / 2 \leq\left(a^{h}, b^{h}\right) \leq 1$ \\
$\mathrm{~B}[L]$ & $a^{l}=K^{l} / L^{l}$ & $b^{l}=H^{l} / L^{l}$ & $2 a^{l} b^{l}=1$ & $1 / 2 \leq\left(a^{l}, b^{l}\right) \leq 1$ \\
$\mathrm{~B}[K]$ & $a^{k}=H^{k} / K^{k}$ & $b^{k}=L^{k} / K^{k}$ & $2 a^{k} b^{k}=1$ & $1 / 2 \leq\left(a^{k}, b^{k}\right) \leq 1$ \\
\hline
\end{tabular}




\subsection{Equilibrium Surface}

The equilibrium state in energy space is confined to some specific surfaces. If we remove the zone restriction, the equilibrium condition (56) can be rewritten as

$$
H=\sqrt{2 L K}, \quad L=\sqrt{2 K H}, \quad K=\sqrt{2 H L} .
$$

They present three parabolic surfaces denoted respectively by $\mathrm{S}[H], \mathrm{S}[L]$ and $\mathrm{S}[K]$. $\mathrm{S}[H]$ stands for the state of vibrating equilibrium, $\mathrm{S}[L]$ for the state of rotating equilibrium and $\mathrm{S}[K]$ for the state of translating equilibrium. Since the zone restrictions are removed, each equilibrium surface covers four phases as showed in Fig. 4. A $1 / 8$ spherical surface $\mathrm{S}[E]$ with the radius $E=\sqrt{H^{2}+L^{2}+K^{2}}$ is called the isoenergy surface.

Taking the example of translating equilibrium surface, we make a general analysis on the equilibrium state. As showed in Fig. 4, the equilibrium surface $\mathrm{S}[K]$ covers four phases, the area in the liquid zone is $\mathrm{S}\left[K^{k}\right]$ (includes the areas of $\mathrm{S}\left[G_{+}^{k}\right]$ and $\mathrm{S}\left[G_{-}^{k}\right]$ ), the area in the solid zone is $\mathrm{S}\left[K^{l}\right]$, and the area in the gas zone is $\mathrm{S}\left[K^{h}\right]$.

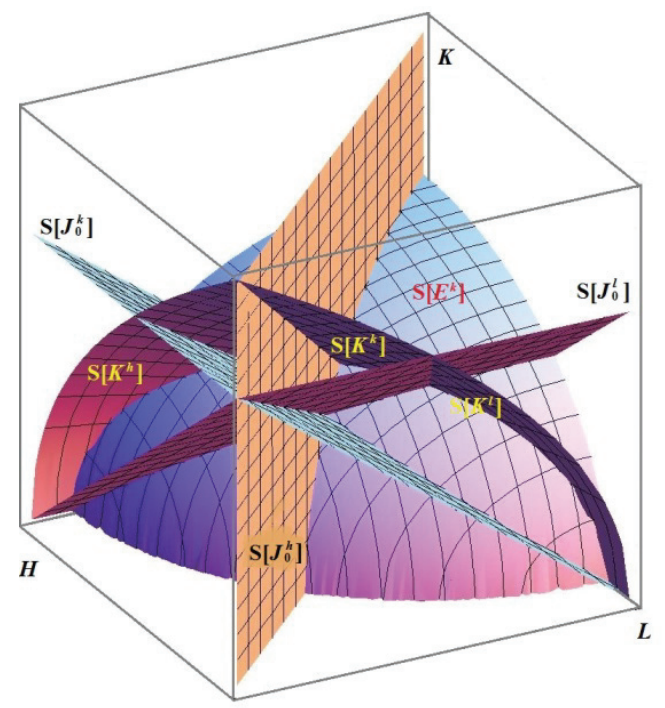

Figure 4. Phase interfaces, translating equilibrium surface and iso-energy surface in energy space.

After removing the zone restriction, the order parameters on the translating equilibrium surface are

$$
a^{k}=H / K, \quad b^{k}=L / K \text {. }
$$

The relationship between the ahead parameter and the back parameter is unchanged $\left(2 a^{k} b^{k}=1\right)$, but the range of the order parameters is no longer confined between $1 / 2$ and 1 . On the area of $\mathrm{S}\left[K^{k}\right], H<K, L<$ $K, 1 / 2<a^{k}<1$ and $1>b^{k}>1 / 2$. S $\left[K^{k}\right]$ represents the stable equilibrium state. On the area of $\mathrm{S}\left[K^{l}\right]$, $L>K>H, a^{k}<1 / 2$ and $b^{k}>1$. S $\left[K^{l}\right]$ represents the excited state of rotation. On the area of $\mathrm{S}\left[K^{h}\right]$, $H>K>L, a^{k}>1$ and $b^{k}<1 / 2$. $\mathrm{S}\left[K^{h}\right]$ represents the excited state of vibration.

According to the permutation symmetry, we can determine the structure of other two equilibrium surfaces. The results are listed in Table 2.

Table 2. Structure of equilibrium surfaces

\begin{tabular}{|c|c|c|c|c|c|c|}
\hline Zone $\backslash$ Phase & $\mathrm{B}\left[G_{+}^{h}\right]$ & $\mathrm{B}\left[G_{-}^{h}\right]$ & $\mathrm{B}\left[G_{+}^{l}\right]$ & $\mathrm{B}\left[G_{-}^{l}\right]$ & $\mathrm{B}\left[G_{+}^{k}\right]$ & $\mathrm{B}\left[G_{-}^{k}\right]$ \\
\hline $\mathrm{S}[H]$ & \multicolumn{2}{|c|}{$\mathrm{S}\left[H^{h}\right]$} & $\mathrm{S}\left[H^{l}\right]$ & & $\mathrm{S}\left[H^{k}\right]$ \\
\hline $\mathrm{S}[L]$ & & $\mathrm{S}\left[L^{h}\right]$ & \multicolumn{2}{c|}{$\mathrm{S}\left[L^{l}\right]$} & $\mathrm{S}\left[L^{k}\right]$ & \\
\hline $\mathrm{S}[K]$ & $\mathrm{S}\left[K^{h}\right]$ & & \multicolumn{2}{c|}{$\mathrm{S}\left[K^{l}\right]$} & \multicolumn{2}{c|}{$\mathrm{S}\left[K^{k}\right]$} \\
\hline
\end{tabular}

To sum up, there are three equilibrium surfaces $(\mathrm{S}[H], \mathrm{S}[L]$ and $\mathrm{S}[K])$ in the energy space. Each surface extends into four phases. The areas of $\mathrm{S}\left[H^{h}\right], \mathrm{S}\left[L^{l}\right]$ and $\mathrm{S}\left[K^{k}\right]$ represent stable states, and other areas 
represent excited states. The three equilibrium surfaces have a total of six stable areas and six excited areas. Each phase contains a stable area and an excited area. The state on the excited area has a tendency to transit to the state of stable area.

\subsection{Interface Discontinuity}

Taking the example of liquid-gas interface $\mathrm{S}\left[J_{0}^{k}\right]$, we make a general analysis on the interfacial property. The plane $\mathrm{S}\left[J_{0}^{k}\right]$ satisfies the conditions $K^{k}=H^{k}$ and $K^{h}=H^{h}$. According to the conditions of two phase equilibrium, $\left(K^{k}\right)^{2}=2 H^{k} L^{k}$ and $\left(H^{h}\right)^{2}=2 L^{h} K^{h}$, we know that the motion energy on the interface satisfy the following relations

$$
K^{k}=H^{k}=2 L^{k}, \quad K^{h}=H^{h}=2 L^{h} .
$$

Above formulas are the conditions for the liquid-gas equilibrium.

By denoting the difference of any quantity $A$ on the interface as $\Delta A^{x y}=A^{y}-A^{x}$, we can calculate the difference of order parameter on the liquid-gas interface.

$$
\begin{gathered}
\Delta a^{k h}=a^{h}-a^{k}=\frac{L^{h}}{H^{h}}-\frac{H^{k}}{K^{k}}=\frac{1}{2}-1=-\frac{1}{2}, \\
\Delta b^{k h}=b^{h}-b^{k}=\frac{K^{h}}{H^{h}}-\frac{L^{k}}{K^{k}}=1-\frac{1}{2}=\frac{1}{2} .
\end{gathered}
$$

The difference of the auxiliary energy can be calculated as

$$
\begin{gathered}
\Delta E^{k h}=E^{h}-E^{k}=\left(L^{h}+K^{h}\right)-\left(H^{k}+L^{k}\right)=3\left(L^{h}-L^{k}\right), \\
\Delta G^{k h}=G^{h}-G^{k}=\left(K^{h}-L^{h}\right)-\left(L^{k}-H^{k}\right)=L^{h}+L^{k}, \\
\Delta Q^{k h}=Q^{h}-Q^{k}=\left(K^{h}+H^{h}\right)-\left(L^{k}+K^{k}\right)=4 L^{h}-3 L^{k}, \\
\Delta J^{k h}=J^{h}-J^{k}=\left(L^{h}-H^{h}\right)-\left(H^{k}-K^{k}\right)=-L^{h} .
\end{gathered}
$$

According to the permutation symmetry, we can determine the change of the order parameters and the auxiliary energies on other two interfaces. The results are concluded in Table 3.

Table 3. The discontinuity at the zone interface

\begin{tabular}{cccc}
\hline Zone interface & Gas-Solid $\mathrm{S}\left[J_{0}^{h}\right]$ & Solid-liquid $\mathrm{S}\left[J_{0}^{l}\right]$ & Liquid-Gas S[Jo $]$ \\
\hline \multirow{2}{*}{ Equilibrium condition } & $H^{h}=L^{h}=2 K^{h}$, & $L^{l}=K^{l}=2 H^{l}$, & $K^{k}=H^{k}=2 L^{k}$, \\
& $H^{l}=L^{l}=2 K^{l}$. & $L^{k}=K^{k}=2 H^{k}$. & $K^{h}=H^{h}=2 L^{h}$. \\
\hline \multirow{2}{*}{ Order parameter } & $\Delta a^{h l}=-1 / 2$, & $\Delta a^{l k}=-1 / 2$, & $\Delta a^{k h}=-1 / 2$, \\
& $\Delta b^{h l}=1 / 2$. & $\Delta b^{l k}=1 / 2$. & $\Delta b^{k h}=1 / 2$. \\
\hline Entire energy & $\Delta E^{h l}=3\left(K^{l}-K^{h}\right)$ & $\Delta E^{l k}=3\left(H^{k}-H^{l}\right)$ & $\Delta E^{k h}=3\left(L^{h}-L^{k}\right)$ \\
\hline Chemical energy & $\Delta G^{h l}=K^{l}+K^{h}$ & $\Delta G^{l k}=H^{k}+H^{l}$ & $\Delta G^{k h}=L^{h}+L^{k}$ \\
\hline Thermal energy & $\Delta Q^{h l}=4 K^{l}-3 K^{h}$ & $\Delta Q^{l k}=4 H^{k}-3 H^{l}$ & $\Delta Q^{k h}=4 L^{h}-3 L^{k}$ \\
\hline Potential energy & $\Delta J^{h l}=-K^{l}$ & $\Delta J^{l k}=-H^{k}$ & $\Delta J^{k h}=-L^{h}$
\end{tabular}

The above results show that the physical quantities on the zone interface are discontinuous. The discontinuity of potential energy predicts the latent energy for the phase transitions across two zones. Also, the order parameter has a $1 / 2$ jump change. This is an important feature of J-type phase transition. Since the G-type phase transition is a transition between two phases within one zone, the change of order parameter must be less than $1 / 2$, and the amount of change is not a fixed value.

\subsection{Mode Factor}

The mode factors $\mu_{1}^{x}, \mu_{2}^{x}, \mu_{3}^{x}$ are defined as the ratio of the entire energy $E^{x}$ to the motion energy $E_{1}^{x}, E_{2}^{x}, E_{3}^{x}$.

$$
\mu_{1}^{x} \equiv \frac{E^{x}}{E_{1}^{x}}=\frac{E^{x}}{H^{x}}, \mu_{2}^{x} \equiv \frac{E^{x}}{E_{2}^{x}}=\frac{E^{x}}{L^{x}}, \mu_{3}^{x} \equiv \frac{E^{x}}{E_{3}^{x}}=\frac{E^{x}}{K^{x}} .
$$

Thus, the entire energy can be expressed in terms of the product of motion energy and mode factor.

$$
E^{x}=\mu_{1}^{x} H^{x}=\mu_{2}^{x} L^{x}=\mu_{3}^{x} K^{x} .
$$

There are three mode factors in each zone, and only one is independent. Taking gas as an example, we express the mode factors in terms of the order parameter. In gas zone, it has $H^{h}>K^{h}, H^{h}>L^{h}$, entire 
energy $E^{h}=L^{h}+K^{h}$, and equilibrium condition $\left(H^{h}\right)^{2}=2 L^{h} K^{h}$ or $2 a^{h} b^{h}=1$. According to the definition of order parameter $a^{h}=L^{h} / H^{h}$ and $b^{h}=K^{h} / H^{h}$, the mode factors are calculated as

$$
\begin{aligned}
& \mu_{1}^{h}=\frac{E^{h}}{H^{h}}=\frac{L^{h}+K^{h}}{H^{h}}=a^{h}+b^{h}=a^{h}+\frac{1}{2 a^{h}}, \\
& \mu_{2}^{h}=\frac{E^{h}}{L^{h}}=\frac{L^{h}+K^{h}}{L^{h}}=1+\frac{b^{h}}{a^{h}}=1+\frac{1}{2\left(a^{h}\right)^{2}}, \\
& \mu_{3}^{h}=\frac{E^{h}}{K^{h}}=\frac{L^{h}+K^{h}}{K^{h}}=\frac{a^{h}}{b^{h}}+1=2\left(a^{h}\right)^{2}+1 .
\end{aligned}
$$

Similarly, the mode factors in solid zone and liquid zone can be obtained. The mode factors in three zones are given below.

$$
\begin{array}{lll}
\mu_{1}^{h}=a^{h}+\frac{1}{2 a^{h}}, \quad \mu_{2}^{h}=1+\frac{1}{2\left(a^{h}\right)^{2}}, & \mu_{3}^{h}=2\left(a^{h}\right)^{2}+1 \\
\mu_{1}^{l}=2\left(a^{l}\right)^{2}+1, \quad \mu_{2}^{l}=a^{l}+\frac{1}{2 a^{l}}, & \mu_{3}^{l}=1+\frac{1}{2\left(a^{l}\right)^{2}} \\
\mu_{1}^{k}=1+\frac{1}{2\left(a^{k}\right)^{2}}, & \mu_{2}^{k}=2\left(a^{k}\right)^{2}+1, & \mu_{3}^{k}=\frac{1}{2 a^{k}}+a^{k} .
\end{array}
$$

\section{Decomposition of Energy}

\subsection{Volume Decomposition}

The motion energies of an object $E_{1}^{x}, E_{2}^{x}, E_{3}^{x}$ can be decomposed by volume $V^{x}$. The factors of volume decomposition are $q_{1}^{x}, q_{2}^{x}, q_{3}^{x}$,

$$
q_{1}^{x} \equiv \frac{H^{x}}{V^{x}}, \quad q_{2}^{x} \equiv \frac{L^{x}}{V^{x}}, \quad q_{1}^{x} \equiv \frac{K^{x}}{V^{x}}
$$

So, the motion energy can be expressed in terms of the product of the volume and the decomposition factor.

$$
H^{x}=q_{1}^{x} V^{x}, \quad L^{x}=q_{2}^{x} V^{x}, \quad K^{x}=q_{3}^{x} V^{x} .
$$

The formula of volume decomposition (65) implies that the volumes of an object in different zones can be different. Since the volume of an object is limited, the volume decomposition is feasible. The factor of volume decomposition is also called energy density. The energy density has the same physical dimension as pressure, but their physical meanings are obviously different.

Each zone has 3 components of energy densities, but only 2 are independent as the equilibrium condition is applied. For example, it has $H^{h}=q_{1}^{h} V^{h}, L^{h}=q_{2}^{h} V^{h}$ and $K^{h}=q_{3}^{h} V^{h}$ in the gas zone. Applying the equilibrium condition $\left(H^{h}\right)^{2}=2 L^{h} K^{h}$, we find the constrain $\left(q_{1}^{h}\right)^{2}=2 q_{2}^{h} q_{3}^{h}$ in the gas zone.

The volume decomposition of auxiliary energy is carried out as follows

$$
\begin{aligned}
& E^{h}=L^{h}+K^{h}=\left(q_{2}^{h}+q_{3}^{h}\right) V^{h}=q^{h} V^{h}, \\
& Q^{h}=K^{h}+H^{h}=\left(q_{3}^{h}+q_{1}^{h}\right) V^{h}=q_{Q}^{h} V^{h}, \\
& J^{h}=L^{h}-H^{h}=\left(q_{2}^{h}-q_{1}^{h}\right) V^{h}=q_{J}^{h} V^{h}, \\
& G^{h}=K^{h}-L^{h}=\left(q_{3}^{h}-q_{2}^{h}\right) V^{h}=q_{G}^{h} V^{h} .
\end{aligned}
$$

where $q^{h}, q_{Q}^{h}, q_{J}^{h}, q_{G}^{h}$ are the density of auxiliary energy. They satisfy following algebraic rules.

$$
q^{h}=q_{2}^{h}+q_{3}^{h}, \quad q_{Q}^{h}=q_{3}^{h}+q_{1}^{h}, \quad q_{J}^{h}=q_{2}^{h}-q_{1}^{h}, \quad q_{G}^{h}=q_{3}^{h}-q_{2}^{h} .
$$

The situation in the liquid and solid zones can be analyzed in a similar way. The conclusions in the three zones are summarized below.

(1). Equilibrium condition expressed by the density of motion energy

$$
\left(q_{1}^{h}\right)^{2}=2 q_{2}^{h} q_{3}^{h}, \quad\left(q_{2}^{l}\right)^{2}=2 q_{3}^{l} q_{1}^{l}, \quad\left(q_{3}^{k}\right)^{2}=2 q_{1}^{k} q_{2}^{k} .
$$

(2). Volume decomposition of the auxiliary energy

$$
\begin{aligned}
& E^{h}=q^{h} V^{h}, \quad E^{l}=q^{l} V^{l}, \quad E^{k}=q^{k} V^{k} . \\
& Q^{h}=q_{Q}^{h} V^{h}, \quad Q^{l}=q_{Q}^{l} V^{l}, \quad Q^{k}=q_{Q}^{k} V^{k} . \\
& J^{h}=q_{J}^{h} V^{h}, \quad J^{l}=q_{J}^{l} V^{l}, \quad J^{k}=q_{J}^{k} V^{k} . \\
& G^{h}=q_{G}^{h} V^{h}, \quad G^{l}=q_{G}^{l} V^{l}, \quad G^{k}=q_{G}^{k} V^{k} .
\end{aligned}
$$

(3). Algebraic rules for the density of auxiliary energy

$$
q^{h}=q_{2}^{h}+q_{3}^{h}, \quad q^{l}=q_{3}^{l}+q_{1}^{l}, \quad q^{k}=q_{1}^{k}+q_{2}^{k} .
$$




$$
\begin{array}{lll}
q_{Q}^{h}=q_{3}^{h}+q_{1}^{h}, & q_{Q}^{l}=q_{1}^{l}+q_{2}^{l}, & q_{Q}^{k}=q_{2}^{k}+q_{3}^{k} . \\
q_{J}^{h}=q_{2}^{h}-q_{1}^{h}, & q_{J}^{l}=q_{3}^{l}-q_{2}^{l}, & q_{J}^{k}=q_{1}^{k}-q_{3}^{k} . \\
q_{G}^{h}=q_{3}^{h}-q_{2}^{h}, & q_{G}^{l}=q_{1}^{l}-q_{3}^{l}, & q_{G}^{k}=q_{2}^{k}-q_{1}^{k} .
\end{array}
$$

\subsection{Particle Decomposition}

The motion energies of an object $E_{1}^{x}, E_{2}^{x}, E_{3}^{x}$ can be decomposed by the number of particles $N$. The factors of particle decomposition are $H_{0}^{x}, L_{0}^{x}, K_{0}^{x}$,

$$
H_{0}^{x} \equiv \frac{H^{x}}{N}, \quad L_{0}^{x} \equiv \frac{L^{x}}{N}, \quad K_{0}^{x} \equiv \frac{K^{x}}{N} .
$$

So, the motion energy can be expressed in terms of the product of the particle number and the decomposition factor.

$$
H^{x}=N H_{0}^{x}, \quad L^{x}=N L_{0}^{x}, \quad K^{x}=N K_{0}^{x} .
$$

The formula of particle decomposition (70) contains the conservational condition of the particle number, $N^{h}=N^{l}=N^{k}=N$. Since the particle number of an object is limited, the particle decomposition is also feasible. The factor of particle decomposition is called energy intensity.

Similar to the way of volume decomposition, we can get conclusions for the particle decomposition.

(1). Equilibrium condition expressed by the intensity of motion energy

$$
\left(H_{0}^{h}\right)^{2}=2 L_{0}^{h} K_{0}^{h}, \quad\left(L_{0}^{l}\right)^{2}=2 K_{0}^{l} H_{0}^{l}, \quad\left(K_{0}^{k}\right)^{2}=2 H_{0}^{k} L_{0}^{k} .
$$

(2). Particle decomposition of the auxiliary energy

$$
\begin{array}{ccc}
E^{h}=N E_{0}^{h}, & E^{l}=N E_{0}^{l}, & E^{k}=N E_{0}^{k} . \\
Q^{h}=N Q_{0}^{h}, & Q^{l}=N Q_{0}^{l}, & Q^{k}=N Q_{0}^{k} . \\
J^{h}=N J_{0}^{h}, & J^{l}=N J_{0}^{l}, & J^{k}=N J_{0}^{k} . \\
G^{h}=N G_{0}^{h}, & G^{l}=N G_{0}^{l}, & G^{k}=N G_{0}^{k} .
\end{array}
$$

(3). Algebraic rules for the intensity of auxiliary energy

$$
\begin{array}{lll}
E_{0}^{h}=L_{0}^{h}+K_{0}^{h}, & E_{0}^{l}=K_{0}^{l}+H_{0}^{l}, & E_{0}^{k}=H_{0}^{k}+L_{0}^{k} . \\
Q_{0}^{h}=K_{0}^{h}+H_{0}^{h}, & Q_{0}^{l}=H_{0}^{l}+L_{0}^{l}, & Q_{0}^{k}=L_{0}^{k}+K_{0}^{k} \\
J_{0}^{h}=L_{0}^{h}-H_{0}^{h}, & J_{0}^{l}=K_{0}^{l}-L_{0}^{l}, & J_{0}^{k}=H_{0}^{k}-K_{0}^{k} . \\
G_{0}^{h}=K_{0}^{h}-L_{0}^{h}, & G_{0}^{l}=H_{0}^{l}-K_{0}^{l}, & G_{0}^{k}=L_{0}^{k}-H_{0}^{k} .
\end{array}
$$

\subsection{Integer Theorem}

The intensity of the major energy $\left(H_{0}^{h}, L_{0}^{l}, K_{0}^{k}\right)$ is consistent with the scale of motion energy $\left(H_{s}, L_{s}, K_{s}\right)$, namely,

$$
H_{0}^{h}=H_{s}=h v, \quad L_{0}^{l}=L_{s}=l z, \quad K_{0}^{k}=K_{s}=k T .
$$

For this reason, $\left(H_{s}, L_{s}, K_{s}\right)$ are also called the scale of major energy.

Now, let's take the example of gas zone to calculate the digits of state vector, motion energy and auxiliary energy under the scale of major energy. In gas zone, it has $H^{h}>L^{h}, H^{h}>K^{h}$, so the state vector can be expressed as

It gives the digit of state vector

$$
\begin{aligned}
\boldsymbol{E}^{h} & =H^{h} \mathbf{i}+L^{h} \mathbf{j}+K^{h} \mathbf{k}=H^{h}\left[\mathbf{i}+\left(L^{h} / H^{h}\right) \mathbf{j}+\left(K^{h} / H^{h}\right) \mathbf{k}\right] \\
& =H_{s} N\left(\mathbf{i}+a^{h} \mathbf{j}+b^{h} \mathbf{k}\right)=H_{s}\left(\widetilde{H}^{h} \mathbf{i}+\widetilde{L}^{h} \mathbf{j}+\widetilde{K}^{h} \mathbf{k}\right)=H_{s} \widetilde{\boldsymbol{E}}^{h} .
\end{aligned}
$$

with the digits of motion energy

$$
\widetilde{\boldsymbol{E}}^{h}=\widetilde{H}^{h} \mathbf{i}+\widetilde{L}^{h} \mathbf{j}+\widetilde{K}^{h} \mathbf{k}
$$

The digits of the auxiliary energy are

$$
\widetilde{H}^{h}=N, \quad \tilde{L}^{h}=N a^{h}<N, \quad \widetilde{K}^{h}=N b^{h}<N .
$$

$$
\begin{array}{ll}
\widetilde{E}^{h}=\widetilde{L}^{h}+\widetilde{K}^{h}=N\left(a^{h}+b^{h}\right), & \widetilde{Q}^{h}=\widetilde{K}^{h}+\widetilde{H}^{h}=N\left(b^{h}+1\right), \\
\tilde{J}^{h}=\widetilde{L}^{h}-\widetilde{H}^{h}=N\left(a^{h}-1\right), & \widetilde{G}^{h}=\widetilde{K}^{h}-\widetilde{L}^{h}=N\left(b^{h}-a^{h}\right) .
\end{array}
$$

In the same way, we conclude the results in three zones as follows.

(1). State vector

(2). Digit of state vector

$$
\boldsymbol{E}^{h}=H_{S} \widetilde{\boldsymbol{E}}^{h}, \quad \boldsymbol{E}^{l}=L_{s} \widetilde{\boldsymbol{E}}^{l}, \quad \boldsymbol{E}^{k}=K_{S} \widetilde{\boldsymbol{E}}^{k} .
$$


(3). Digit of motion energy

$$
\begin{aligned}
\widetilde{\boldsymbol{E}}^{h} & =\widetilde{H}^{h} \mathbf{i}+\widetilde{L}^{h} \mathbf{j}+\widetilde{K}^{h} \mathbf{k}, \\
\widetilde{\boldsymbol{E}}^{l} & =\widetilde{H}^{l} \mathbf{i}+\widetilde{L}^{l} \mathbf{j}+\widetilde{K}^{l} \mathbf{k}, \\
\widetilde{\boldsymbol{E}}^{k} & =\widetilde{H}^{k} \mathbf{i}+\widetilde{L}^{k} \mathbf{j}+\widetilde{K}^{k} \mathbf{k} .
\end{aligned}
$$

$$
\begin{gathered}
\widetilde{H}^{h}=N, \quad \tilde{L}^{h}=N a^{h}<N, \quad \widetilde{K}^{h}=N b^{h}<N . \\
\widetilde{H}^{l}=N b^{l}<N, \quad \widetilde{L}^{l}=N, \quad \widetilde{K}^{l}=N a^{l}<N . \\
\widetilde{H}^{k}=N a^{k}<N, \quad \widetilde{L}^{k}=N b^{k}<N, \quad \widetilde{K}^{k}=N .
\end{gathered}
$$

(4). Digit of auxiliary energy

$$
\begin{array}{ccc}
\tilde{E}^{h}=N\left(a^{h}+b^{h}\right), & \tilde{E}^{l}=N\left(a^{l}+b^{l}\right), & \tilde{E}^{k}=N\left(a^{k}+b^{k}\right) . \\
\tilde{Q}^{h}=N\left(b^{h}+1\right), & \tilde{Q}^{l}=N\left(b^{l}+1\right), & \tilde{Q}^{k}=N\left(b^{k}+1\right) . \\
\tilde{J}^{h}=N\left(a^{h}-1\right), & \tilde{J}^{l}=N\left(a^{l}-1\right), & \tilde{J}^{k}=N\left(a^{k}-1\right) . \\
\tilde{G}^{h}=N\left(b^{h}-a^{h}\right), & \tilde{G}^{l}=N\left(b^{l}-a^{l}\right), & \tilde{G}^{k}=N\left(b^{k}-a^{k}\right) .
\end{array}
$$

It can be seen that the digital state can be completely determined as long as the number of particles $N$, the order parameter $a$ (or $b$ ) are known.

The amount of particles in a thermodynamic system is enormous. Using the scale of major energy as the unit of energy has a sufficiently high resolution. If the digits of motion energy take positive integers, then the digits of all auxiliary energy are integers, too. This conclusion is called the integer theorem of energy.

\subsection{Digital State}

The integer combination of energy digits $(\widetilde{H}, \widetilde{L}, \widetilde{K}, \widetilde{E})$ is called the digital state. The digit of entire energy $\tilde{E}$ is called energy level. The digital states are discrete points that satisfy the integer theorem on the equilibrium surface. The interval between two adjacent digital states is called the energy gap. The distribution of the digital state on the equilibrium surface is uneven, and the gaps of energy vary in different directions.

The digital state can be easily calculated by the equilibrium condition. On the rotating equilibrium surface, for example, the equilibrium condition is $\widetilde{L}^{2}=2 \widetilde{K} \widetilde{H}$ and the entire energy is $\widetilde{E}=\widetilde{K}+\widetilde{H}$. Table 4 lists the digital states in the cases of $\widetilde{H}=1,2,3$. In table $4, S\left[L^{k}\right]$ is excited state, $L\left[J_{0}^{l}\right]$ is the intersecting line of $\mathrm{S}[L]$ and $\mathrm{S}[K], L\left[J_{0}^{h}\right]$ is the intersecting line of $\mathrm{S}[H]$ and $\mathrm{S}[L]$. These digital states can explain the spectral lines of the hydrogen atoms and the helium ions [4].

Table 4. Digital state on the surface of rotating equilibrium

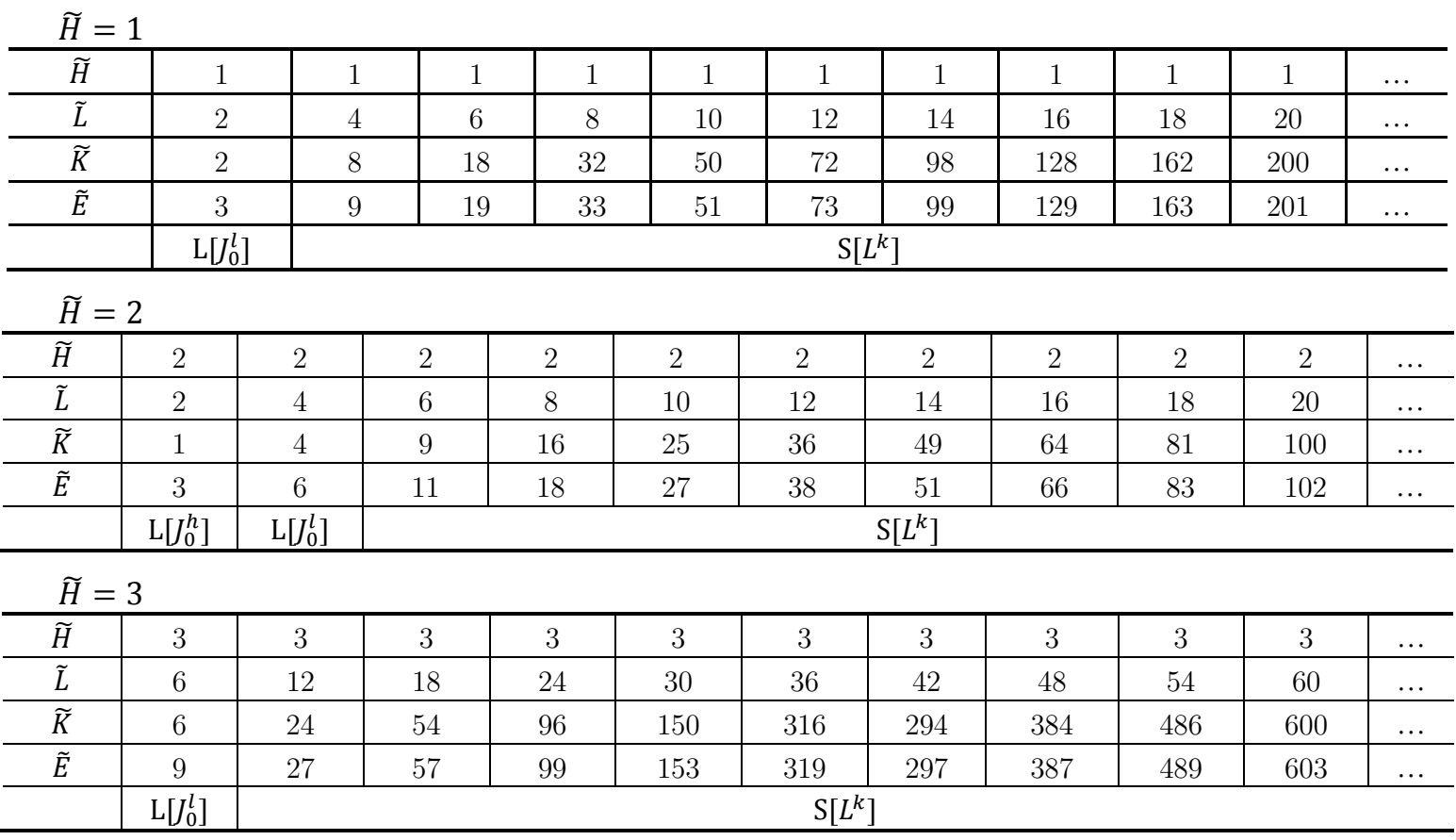




\subsection{Equations of State}

The entire energy can be decomposed as follows

$$
\begin{aligned}
& E^{h}=\mu_{1}^{h} H^{h}=\mu_{1}^{h} N H_{s}=N E_{0}^{h}, \\
& E^{l}=\mu_{2}^{l} L^{l}=\mu_{2}^{l} N L_{s}=N E_{0}^{l}, \\
& E^{k}=\mu_{3}^{k} K^{k}=\mu_{3}^{k} N K_{s}=N E_{0}^{k} .
\end{aligned}
$$

So, the intensity of entire energy can be expressed by the scale of major energy as

$$
E_{0}^{h}=\mu_{1}^{h} H_{s}, \quad E_{0}^{l}=\mu_{2}^{l} L_{s}, \quad E_{0}^{k}=\mu_{3}^{k} K_{s} .
$$

Let the volume decomposition $E^{x}=q^{x} V^{x}$ and particle decomposition $E^{x}=N E_{0}^{x}$ be equal, we obtain the following equations,

$$
\begin{gathered}
E^{h}=q^{h} V^{h}=\mu_{1}^{h} N H_{s}, \\
E^{l}=q^{l} V^{l}=\mu_{2}^{l} N L_{s}, \\
E^{k}=q^{k} V^{k}=\mu_{3}^{k} N K_{s},
\end{gathered}
$$

where $H_{s}=h v, L_{s}=l z, K_{s}=k T$. Formula (81) is named as the equations of state. It contains three zones, which are applicable to gas state, solid state and liquid state, respectively.

\section{$7 \quad$ System of Scales}

\subsection{Scale, Unit and Dimension}

The scale is a key concept in the theory of real physics. The first function of scale is the identification of a physical quantity, which is an extension of the concept of physical dimension. The second function of scale is the quantification standard, which is an extension of the concept of measure units. The difference between dimension and scale is that the former does not have the second function of the latter. The difference between unit and scale is that the former is a fixed standard and the latter is a free variable. A physical quantity has only one scale, but it can have different units.

The system of units is a set of fixed standards for the measurement of physical quantities. For example, the International System of Units (SI system) defines seven units of measure as a basic set from which all other SI units can be derived. The SI base units and their physical quantities are the meter for length, the kilogram for mass, the second for time, the ampere for electric current, the kelvin for temperature, the candela for luminous intensity, and the mole for amount of substance. The SI base units form a set of mutually independent dimensions as required by dimensional analysis commonly employed in science and technology.

The system of scales is a set of free variables for the identification of physical quantities. For examples, in this article, $r_{s}$ represents the scale of length, $t_{s}$ represents the scale of time, $M_{s}$ represents the scale of mass, $p_{s}$ represents the scale of momentum, and $E_{s}$ represents the scale of energy. There are only three independent base scales, and all other scales can be derived from the base scales. Different combination of base scales makes up different system of scales. Different system of scales would lead to different physical theories and will seriously affect the understanding of physical concepts such as time, space, mass and energy.

\subsection{Newton Scale System}

The scale system based on length, time and mass is called Newton scale system. The base scales in Newton scale system are $r_{s}, t_{s}, M_{s}$. The main derived scales are

$$
\begin{gathered}
u_{s}=r_{s} / t_{s}, \quad p_{s}=M_{s} r_{s} / t_{s}, \quad E_{s}=M_{s}\left(r_{s} / t_{s}\right)^{2}, \\
\omega_{s}=1 / t_{s}, \quad I_{s}=M_{s} r_{s}^{2}, \quad s_{s}=M_{s} r_{s}^{2} / t_{s} .
\end{gathered}
$$

The definition of Newton scale system is consistent with Newton's viewpoint that space, time and mass are independent of each other.

\subsection{Einstein Scale System}

If time, speed and energy are taken as the base quantities with the scales $t_{s}, u_{s}, E_{s}$, the main derived scales are 


$$
\begin{gathered}
r_{s}=u_{s} t_{s}, \quad M_{s}=E_{s} / u_{s}^{2}, \quad p_{s}=E_{s} / u_{s}, \\
\omega_{s}=1 / t_{s}, \quad I_{s}=E_{s} t_{s}^{2}, \quad s_{s}=E_{s} t_{s} .
\end{gathered}
$$

In the theory of relativity, Einstein fixed the scale of velocity with the speed of light, i.e. $u_{s}=c$, so the derived scales becomes

$$
\begin{array}{ll}
r_{s}=c t_{s}, & M_{s}=E_{s} / c^{2}, \quad p_{s}=E_{s} / c, \\
\omega_{s}=1 / t_{s}, & I_{s}=E_{s} t_{s}^{2}, \quad s_{s}=E_{s} t_{s} .
\end{array}
$$

Einstein's viewpoints are that time and space are dependent (according to Lorentz transformation), mass and energy are equivalent (according to $E=M c^{2}$ ). This concept is based on $t_{s}, c, E_{s}$ as the base scales, so this system of scales is called Einstein scale system. Our theory requires that space and time must be independent of each other, even though there is a relation $\left(r_{s}=c t_{s}\right)$ between the scales of space and time. Both scale and digit of the mass allow change, but their product must remain invariant to maintain the conservation of mass. The scale relation of mass and energy $\left(E_{s}=M_{s} c^{2}\right)$ cannot be interpreted as the conversion between mass and energy.

\subsection{Quantum Scale System}

Taking $u_{s}, s_{s}, \omega_{s}$ as base scales and choosing $u_{s}=c, s_{s}=h, \omega_{s}=v$, the derived scales are

$$
\begin{gathered}
t_{s}=1 / v, \quad r_{s}=c / v, \quad E_{s}=h v, \\
M_{s}=h v / c^{2}, \quad I_{s}=h / v, \quad p_{s}=h v / c .
\end{gathered}
$$

As $c$ and $h$ are constants, $v$ is the only free scale. Above scales show the common quantum relation, which is called a quantum scale system. $r_{s}=c / v$ indicates that the wavelength is the scale of length. $r_{s} p_{s}=h$ and $E_{s} t_{s}=h$ give the strict explanation to the Heisenberg's uncertainty relations.

\section{Conclusions}

An object has three spatial states in three-dimensional space: position, posture and profile. The change of the spatial states corresponds to three modes of motion: translation, rotation and vibration. The energy of three modes of motion constitutes a Cartesian energy space. The energy space can be divided into three zones and six phases. The three zones correspond to the three states of object (liquid, solid and gas). There are three parabolic surfaces representing the equilibrium state of object. The state transition and the phase transition are explained and analyzed according to the structure of the energy space. Strict analysis proves that the digits of energy in equilibrium state must be integers. The Planck constant and Boltzmann constant are both scale transformation coefficients of the motion energy. It is concluded that the essence of quantum is no other than the scale.

Acknowledgements. This research is partially supported by NSFC (No.61775102). The author sincerely thanks Professor Francis T. S. Yu for his strong support and inspiring discussion. The author also thanks Mr. Ning Tian for his constant encouragement and enthusiastic assistance in many ways.

\section{References}

1. S.N. Jin and Y.L. Ma, Theoretical Mechanics, Higher Education Press, Beijing. 2002.

2. S.H. Guo, Electrodynamics, Higher Education Press, Beijing. 2008.

3. J.Y. Zeng, Quantum Mechanics, Science Press, Beijing. 2018.

4. Z.C. Liang, Physical principles of finite particle system, Scientific Research Publishing, Wuhan. 2015.

5. Z.C. Liang, "Essence of light: particle, field and interaction". in Proc. SPIE (Optics+Photonics, San Diego). 2018. 10755, 1075501-10755014.

6. S.Z. Li, Linear Algebra, Higher Education Press, Beijing. 2006.

7. L.D. Landau and E.M. Lifshitz, Theory of Elasticity (3rd ed.), Butterworth Heinemann. 1986.

8. Z.H. Lin, Thermodynamics and Statistical Physics, Peking University Press, Beijing, 2007. 